\title{
COMPARING DESIGN OUTCOMES ACHIEVED BY TEAMS OF EXPERT AND NOVICE DESIGNERS THROUGH AGENT- BASED SIMULATION
}

\author{
Singh, Harshika (1); \\ Cascini, Gaetano (1); \\ McComb, Christopher (2) \\ 1: Politecnico di Milano; \\ 2: The Pennsylvania State University
}

\begin{abstract}
Previous research has shown that experienced and novice individuals behave and think differently. Although experienced individuals are better at solving problems, organisations are constantly forming teams of experienced and novice designers to work together on their projects. It is crucial to understand how these teams affect the design outcomes. Therefore, the aim of the study is to investigate how collaborative design teams perform when composed of varying numbers of experienced and novice agents. Specifically in this paper, teams work on a routine task and design outcomes are measured in terms of quality, variety, and exploration of the design space. Since the parameters in the empirical laboratory experiments are difficult to control, an agent-based model was used to simulate these teams. In general, the results show that a team of novice agents with a small number of experienced agents produces solutions of higher quality than an all novice team of agents. However, an all novice team of agents does provide a higher variety of solutions. These results have important implications when teams of experienced and novice designers are formed to work together in practice.
\end{abstract}

Keywords: Collaborative design, Teamwork, Computational design methods, Human behaviour in design, Design process

\section{Contact:}

Singh, Harshika

Politecnico di Milano

Department of Mechanical Engineering

Italy

harshika.singh@polimi.it 


\section{INTRODUCTION}

Design teams are often composed of individuals with varying levels of expertise. Though having a diversity of experiences within a team does add to the innovation potential of the team, the varying level of experience among the team members can influence design team dynamics and may give rise to power hierarchies. Experienced individuals are often more influential as they are confident and may affect learning and decision-making in novices in the team (Chamorro-Koc et al., 2009; Klucharev et al., 2008). On the other hand, teams in which there are no experienced individuals may still give rise to certain 'influential' individuals who may drive the team process (Singh et al., 2020). Hence, making collaboration design is more complex to study than 'individual design' as the interaction between the team members affects individuals' cognitive and social abilities. The design project results depend on the combination and interaction among the team members. Although much research in the past has been done to compare the skills and approaches of novices and experienced practitioners, more attention is required to see the effect of social interactions on the design outcomes when addressing a routine design task. Though the term expert and experience are slightly different (as the former is used in the context of having extensive knowledge in one's domain while the latter is based on time, where if one does a task for a long time, it becomes easier), they have been used interchangeably in the paper. This is because there is a huge overlap in the two terms as one can become an expert simply by working on a task for a lot of time.

Since studying interactions and social factors is difficult to control in laboratory experiments due to their dynamic nature, the paper uses the MILANO (Model of Influence, Learning, and Norms in Organizations) framework, originally presented by Singh et al. (2020a, 2020b) to study this phenomenon. MILANO models collaborative design teams, thus providing a platform to simulate various team compositions and see their effect on design outcome.

In addition to further validating the model's functionality by comparing the findings presented in this paper with the literature, the paper also offers insights into design team behaviour. It shows how experience-novice team composition affect design outcome with respect to the nature of the design task. This provides a possibility to exploit the results to implement proper team management strategies to extract the full creative potential of the design teams.

The structure of the paper consists of background literature, followed by a brief description of the model and method used for obtaining the results. The paper ends with a section presenting and discussion of the results that is followed by a conclusion.

\subsection{Background}

The impact of experience on design creativity outcome, performance and design products has been studied in the past (Chen, 2001). Some researchers studied how mental models of experienced designers affected their way of approaching a design task (Chamorro-Koc et al., 2009) while others worked on the cognitive actions of novices and experienced individuals (Cross, 2004). It is clear from the studies that novices and experienced individuals have different approaches towards the design task. While experienced individuals use their strategies to solve the problem (Ahmed et al., 2003), novices are unaware of these strategies employed by the experienced individuals (Ahmed and Wallace, 2004) and often use a trial-and-error approach (Ahmed et al., 2003). Most of the past literature has studied novice or experienced individuals on an individual basis, so little is known about the influence of interaction between the two when placed in the same team. Singh and Casakin (2018) also proposed an agent-based framework where experts and novices in a design team might use the analogy that influences team cohesion and team collaboration. Some agent-based models have been built to study how novice-novice, expert-expert and novice-expert scrum team pairs affect productivity (Wang, 2018) or similar links affect collaboration network and team performance (Guimerà et al., 2005). Others like Perisic et.al. (2019) have investigated their impact on the exploration of problem-solution space.

There are several components that affect the creative process such as individual cognitive abilities, personality characteristics and social factors. A study by Georgilas et al. (2019) showed that professional practitioner has an impact on the learning process and the delivery from the novices (students), hence negatively affects the creative potential of open-ended projects. Another study showed that adding a professional to student teams did not increase creativity but had a major influence on the selected functions (Zeiler, 2013). Novices and experienced individuals contribute differently, and one should consider the interaction between them. The interaction among individuals 
in a group gives rise to social influence that affects learning and decision making. It was found that social influence not only affects people's willingness to adopt opinions but also the norms of other more influential people (Zaki et al., 2011). As experienced individual exhibit more confidence (Chamorro-Koc et al., 2009) and the qualities of a leader (Germain, 2012), they might have more influencing power. Hence, having novices and experienced individuals in the same design team affect learning (especially during idea generation) and decision-making (especially when selecting ideas) in novices as they might value experienced opinion more than their fellow novice team members (Meshi et al., 2012). Novices gather knowledge from the experienced members of the team and use it to their existing knowledge to generate new solutions (Deken et al., 2012). Even a short exposure to an experienced individual can lead novices to follow them (Klucharev et al., 2008). The noviceexperienced interactions in a team could be considered highly complex (Deken et al., 2009) and not much attention has been given when evaluating design team outcomes.

As seen from the above literature, most prior work has studied individuals (i.e., either experienced or novices) and the effect of having novices and experienced individuals in the same flat team (a team where there is no organisational hierarchy and everyone could contribute) has not been explored. It is also known that the nature of the task given to novice and experienced participants affects the performance (Haerem and Rau, 2007; McComb et al., 2015). In other words, team processes and outcomes depend on the nature of the task given to the participants. In the context of this paper, the complexity of a task is defined by the ease of finding an above-average solution. In order to understand the effect of these different team composition of novice and experienced agents on design outcomes when working on a difficult task (that has fewer or one alternative solution thus hard to find solutions) or a simple easy task (multiple alternative solutions hence easy to find), the following research question is identified:

How are design outcomes affected by experience-differentiated team compositions?

The design outcome in the context of the paper is measured through a combination of solution quality, variety, and exploration. Therefore, the main contribution of the work is to design engineering research as it explores how different experience-novice team composition affects design outcomes in terms of quality, exploration and variety. The model considers the effects of social interactions between novices and experienced individuals when placed together in a team. Furthermore, it provides an agent-based framework for studying collaborative design teams at a fine-grained level that could be used by future researchers for studying other aspects of design team collaboration. The following section briefly describes the model.

\section{MODEL DESCRIPTION}

The co-design process simulated in the agent-based model is shown in Figure 1. Emulating real codesign practice, it starts in the form of a project with multiple sessions of collaborative idea generation and idea selection. At the end of each session the final solution is proposed to the controller agent, which is equivalent to project controller, leader, manager or others in similar roles. The feedback from the controller agent helps agents to learn and accordingly propose solutions in the following session.

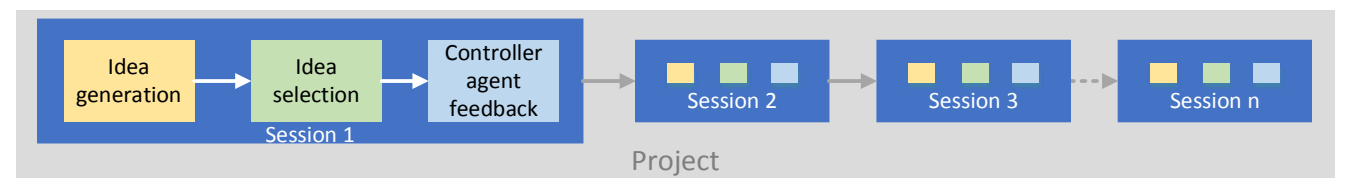

Figure 1. Model simulation flow

The collaborative design activity starts with a team of agents working on a design task to produce solutions that are acceptable by the controller agent. The design task/problem as shown as an example in Figure 2, is computationally represented as a 2-dimensional function where each dimension denotes a design aspect (in this case 2 aspects were considered). Any point on the design space is the potential solution and can have a value between $0-1$ (solution value $=1$ in lightest hue and worst solution value $=0$ in darkest hue) (Singh et al., 2020a). The results of the design outcome presented in the paper are related to the design space with 1 and 5 peaks where peaks denote the best alternative options. The 1 best solution or peak could be considered equivalent to a conceptual design problem where it is difficult to find solutions while 5 best solutions or peaks as a design problem where it is easy to find solutions. In other words, the number of peaks is analogous to the ease of finding a good solution for a 
conceptual design problem. The curvature of the peaks (steep or curved) is analogous to the refinement or optimisation of a detail design activity (kept constant for the results presented in this paper).

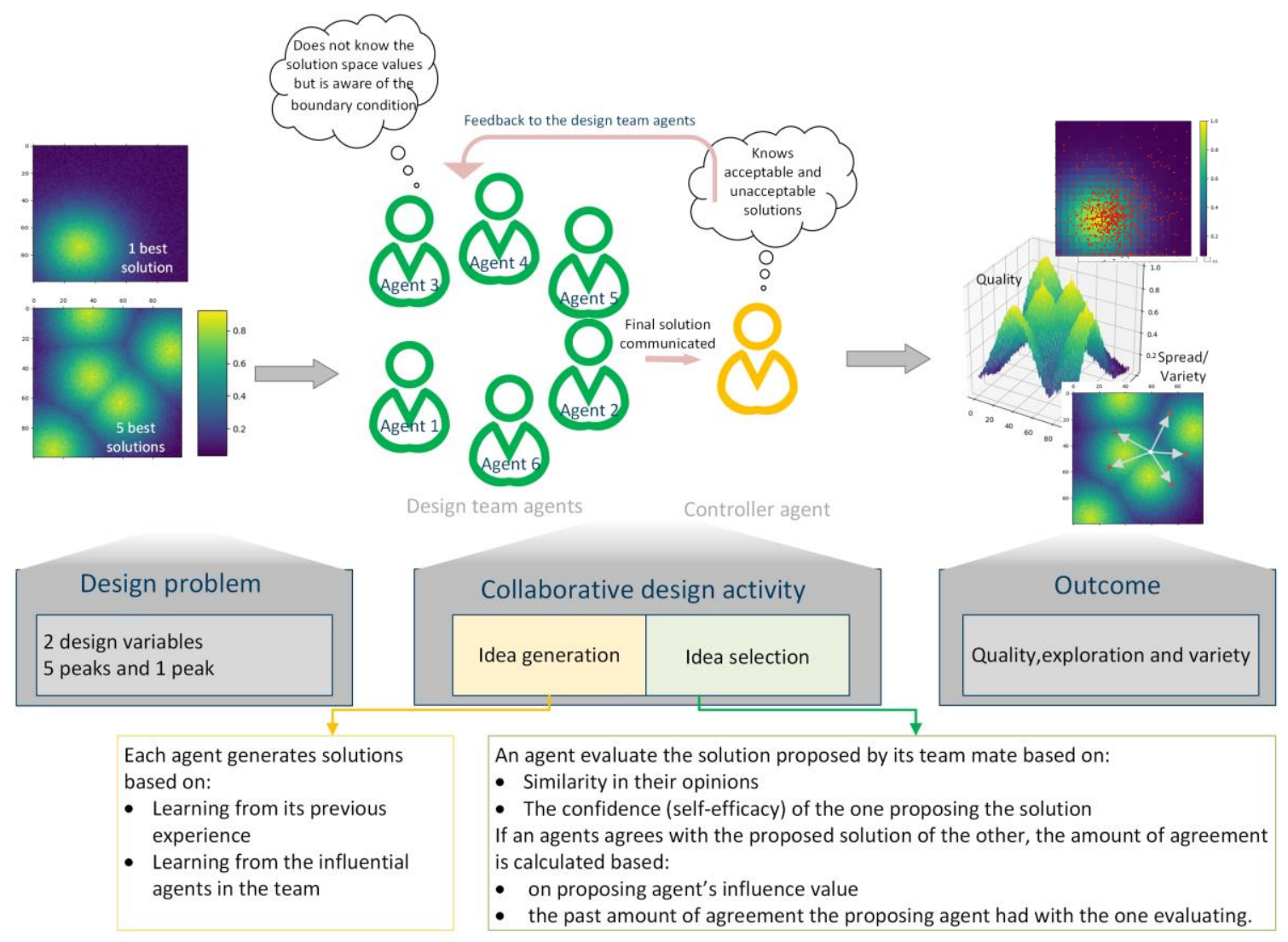

Figure 2. Model framework

A team of design agents work on the design problem throughout a project. Agents here are individuals human who can be novices or with experienced individuals. A novice agent is referred to in this paper as someone who lacks exposure to similar problems or tasks as that of one, he/she is currently working. Whereas an experienced agent is the one who has encountered similar tasks before, therefore has knowledge of failure or error points. The minds of individuals with prior knowledge work differently than those of the newcomers. For example, an experienced individual could see the design problem as well as the possible procedures for solving the problem. Similarly, in the model, an experienced agent has a tendency to work from its past known areas to solve the current unknowns as it has worked on similar problems and could recall those experiences when working on the current one. Novices, on the other hand, work on the current unknown problem and use trial and error techniques to solve it. Since novices also lack the knowledge of failure point or zones when solving a problem, they take more time to reach a satisfactory solution. The flowchart in Figure 3 shows how experienced agents were created and a team of novice-experience agents were made.

The agents generate solutions during the idea generation based on the given set of rules as described in (Singh et al., 2020a). Each agent has its way to explore solution space based on its energy which decreases with the length of an idea generation session. An agent has memory to store its past event in the form of positive and negative experiences. These past experiences of the previous sessions are recalled, and an agent learns from them by avoiding the area of the failures on the solution space and moving in the direction of the past success. Learning from its peers depends on the influence value it has from the other agent. Influence value is calculated based on the self-efficacy and trust between the two agents. Trust on the other hand is based on an agent's reputation (computed as the ratio of the number of accepted solutions to the total number of proposed solutions) and familiarity (computed as the number of sessions the two agents have in common). An agent will be drawn towards the most influential agents in its team than its own success if it has low self-efficacy. As the trust and selfefficacy levels of an agent are dynamic and change with the sessions, the influencers may not be constant. 


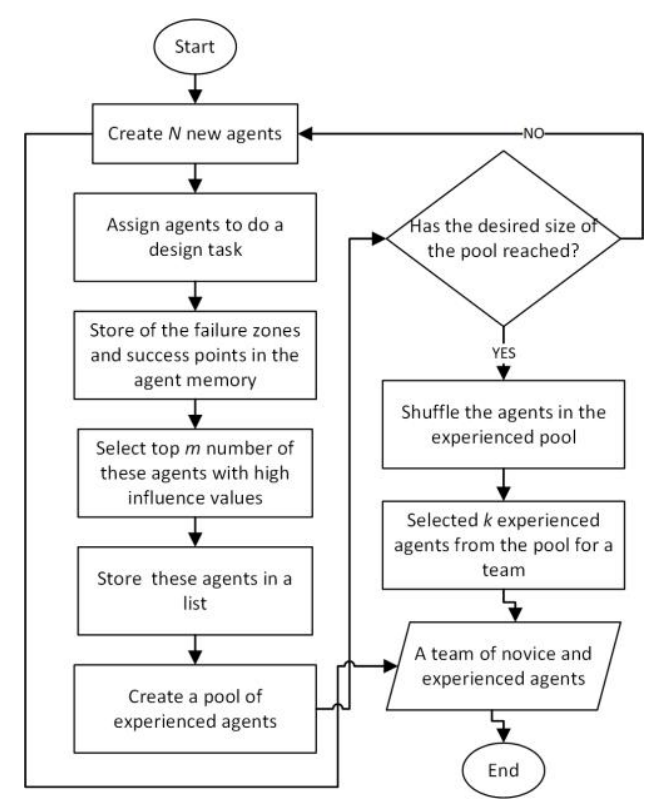

Figure 3. A flowchart showing the creation of novice-experience agent teams

Idea selection follows idea generation as seen in Figure 2 (Singh et al., 2020b). In idea selection, the agents propose their solutions to the team and decide which final solution to communicate to the controller agent. The probability of an agent to be selected to propose its solution depends on how the self-efficacy is distributed in the team. In other words, an agent with high self-efficacy has a higher probability to communicate than someone with lower. Similar to the real-world brainstorming session where similar ideas are combined, the agents in the model also combine their similar proposed solutions. The computational similarity between the solutions is defined by the distance between the solution points on the design space. In other words, two solutions are similar if they are close to each other on a solution space, and dissimilar if far apart. However, the decision-making in individuals could be affected by the presence of a highly confident individual in the group as well as the majority effect "caused by the presence of a critical mass of laypeople sharing similar opinions" (Moussaild, et al., 2013). This gives rise to coalition groups in teams as the opinions of individuals that are close to each other tends to dominate the group judgment process. The cumulative self-efficacy of these coalition groups is a major factor that decides the amount of agreement a team has on the proposed solutions. The solutions with the maximum agreement are communicated to the controller agent. Depending on the quality of the solution proposed, the controller agent provides relevant feedback to the team.

\section{METHOD DESCRIPTION}

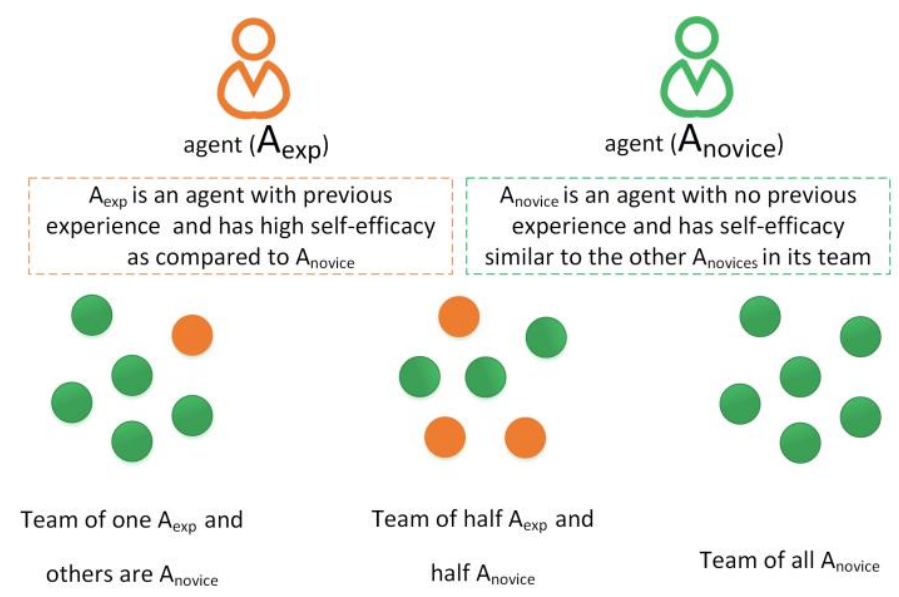

Figure 4. Three cases were framed to explore the research question

There are several parameters present during a collaborative activity (Singh et al., 2019), however only the ones relevant to the goal of the paper were considered. Parameters like team size, the number of 
sessions, the number of experienced agents, number of novices and design task were assigned and kept constant throughout the simulation. At the beginning of the simulation self-efficacies of the agents were controlled (i.e., each agent was allotted self-efficacy), the agent self-efficacy along with familiarity, trust and reputation are dynamic and change with sessions. Except for the experienced agents, all the other novice agents do not have any previous experience of working on the given task.

To explore the research question, three scenarios were framed and tested (see Figure 4). The first scenario tested the situation when the team has only one experience agent with novices. The second case is when half of the team is experienced and the other half is novice, and the last case is when all the agents in a team are novice. All of the novice agents have similar self-efficacy when they start working on a design task while the experienced agents have higher self-efficacy.

Computationally the design task outcome is measured in three ways that could be referred in terms of design creativity as the quality (i.e., the utility value of the solution), exploration and the variety of the solutions. These three different measures were chosen as it would be useful to see how much the agents explore the design space when generating solutions while considering the quality and diversity of their final solutions that were proposed to the controller agent.

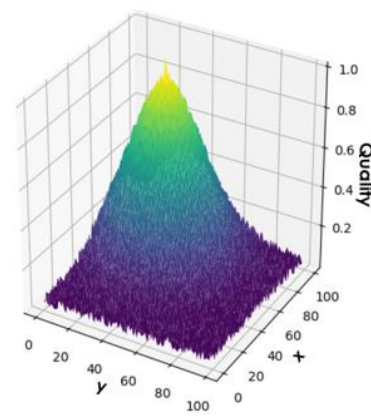

1) Quality

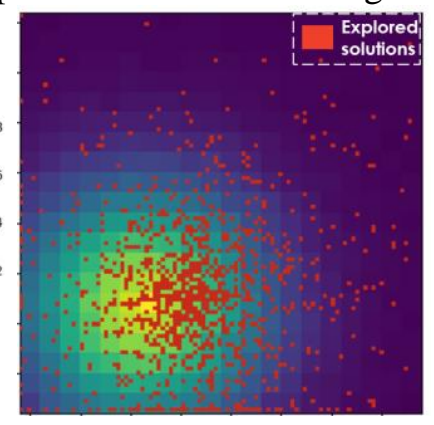

2) Exploration Index (EI)

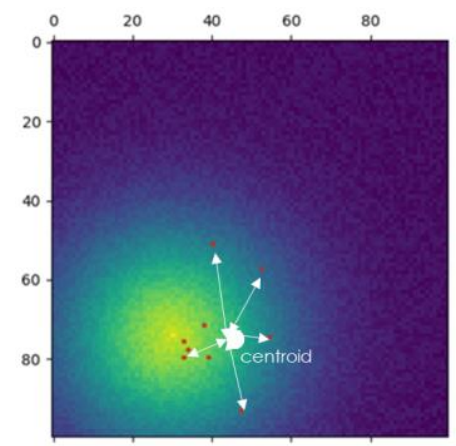

3) Spread of the solutions

Figure 5. Design outcome measuring indices

1. Quality: The quality of the solution is the value of a point on a design solution space. As seen from Figure 5.1 that the highest value of the quality is 1 and the lowest is 0 . The quality values accessed here are of the final solutions proposed by the teams to the controller agent, i.e., one after each session throughout the project.

2. Exploration index: The exploration index is the number of points (solutions) explored when generating solutions on a lower resolution solution space $\left(\operatorname{Soln}_{\text {lowr }}\right)$ to the area of this lower resolution space $\left(A r e a_{\text {lowr }}\right)$, an example can be seen in Figure 5.2 and is given in Equation 1. The lower resolution of solution space means that the original solution space $(100 \times 100$ units $)$ is decreased in size by a factor ( 5 in this case), so that the resultant is a smaller space $(20 \times 20$ units). This means that if an agent explores solutions within 5 units of neighbouring cells, it is counted as one-unit exploration. It was done to avoid having an inaccuracy that could arise; e.g., when an agent explores immediate neighbour cells to an agent exploring 4 cells at a lager unit distance.

$$
E I=\text { Soln }_{\text {lowr }} / \text { Area }_{\text {lowr }}
$$

3. Spread: The spread of the solutions tells how disperse are the solutions from each other. Spread in other words is analogous to the variety of the solutions (given in Equation 2). Here the spread (an example can be seen in Figure 5.3) is measured for the final proposed solutions by the team to the controller agent.

If $S$ is a set of $n$ proposed solutions, $S=\left\{\left(x_{1}, y_{1}\right),\left(x_{2}, y_{2}\right), \ldots,\left(x_{n}, y_{n}\right)\right\}$. The coordinates of a centroid $c=\left(c_{1}, c_{2}\right)$, are calculated as $\left(c_{1}, c_{2}\right)=\left(\frac{1}{n} \sum_{i=1}^{n} x_{i}, \frac{1}{n} \sum_{i=1}^{n} y_{i}\right)$. The average distance $\mu$ from that centroid is $\mu=\frac{1}{n} \sum_{i=1}^{n}\left\|S_{i}-c\right\|$, where $\left\|S_{i}-c\right\|$ is the Euclidean distance $d(d=$ $\left.\sqrt{\left(x_{i}-c_{1}\right)^{2}+\left(y_{i}-c_{2}\right)^{2}}\right)$. The spread or the variety among the solutions can be calculated as the standard deviation of these distances from the centroid (as given below). Where $N$ is the total number of distances between the solution coordinates and the centroid. 


$$
\text { Spread }=\sqrt{\frac{1}{N} \sum_{j=1}^{N}\left(d_{j}-\mu\right)^{2}}
$$

\section{RESULTS AND DISCUSSION}

\subsection{Quality}

The simulation results presented are based on the Monte Carlo method to reduce the uncertainty in the results. Figure 6 (left) shows that a team of half experienced and half novices produces the best solution quality and teams of all novices do the worst. This is likely observed because novices socially integrate with experienced individuals in teams, they tune their activities on the levels of experienced individuals (Fronza et al., 2011). Similar results were found in Atman et al. (1999) where novices did not produce quality design solutions. This could be because of the obvious reason that the experienced agents are aware of the failure zones and success points of the design problem. Experienced agent teams (both 1 and 3 experienced agents) behave similar to those of the experienced individuals in the real- world who start solving a problem from a 'higher place' or start the session with superior quality because of their ability to recall meaningful information before beginning the current task (National Research Council, 2000).
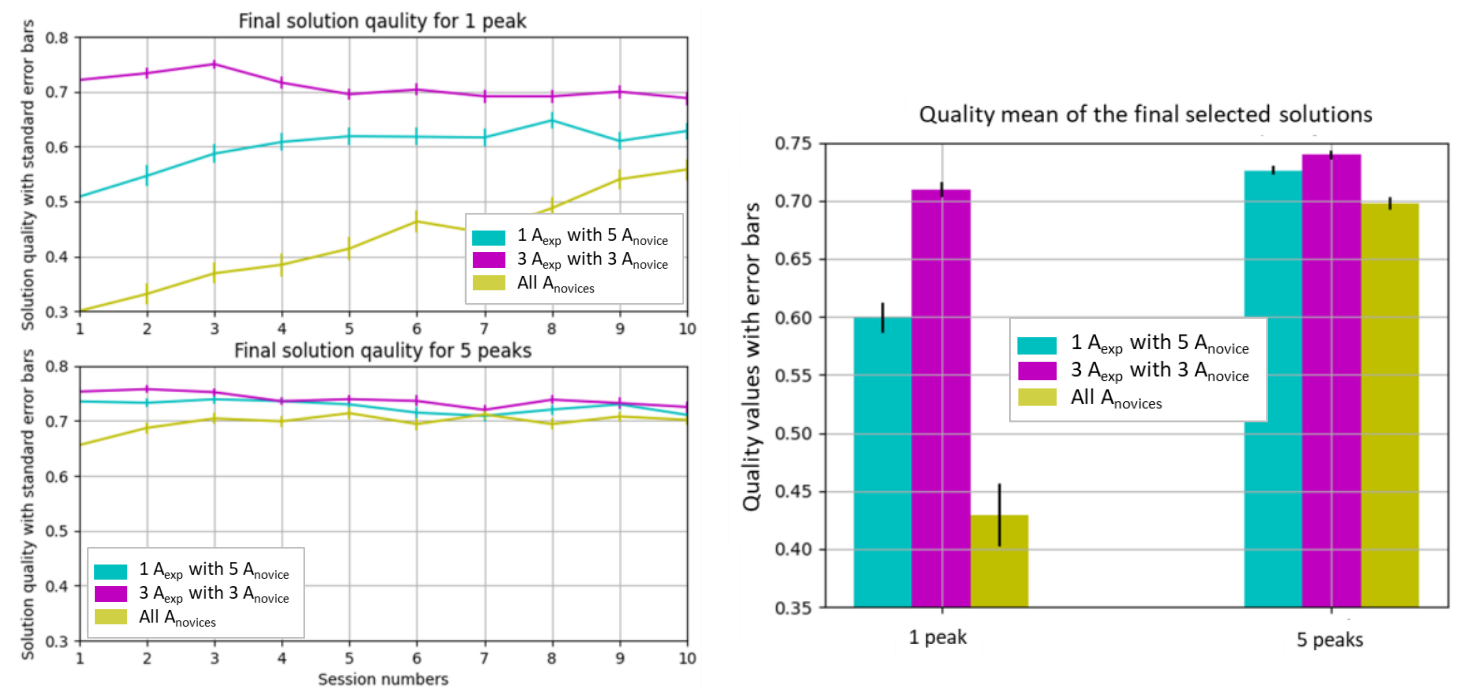

Figure 6.(left) Final solution quality of the solutions session-wise for one best solution (top) and multiple solutions (bottom); (right) overall final solution quality for one best solution and multiple best solution problems

Though the solution quality of the final solutions by the experienced agent(s) in teams with novices is constantly better, it gets stable after some sessions, while all novice team continuously improves their solution quality. As the experienced agent teams ( 1 and 3 experienced with novices) immediately start producing above-average solutions, hence they receive good feedback from the controller agent. This makes the team propose solutions close to the previous ones which result in less change in the quality after some sessions. This behaviour indicates 'fixation' as found by Ball et al. (1994) in their study. The agents in the all novice team behave differently when the tasks become difficult (i.e., one peak), they take more time (sessions) to reach an above-average solution (Perisic et al., 2019), hence a gradual improvement in their solution quality.

Figure 6(right) shows that a larger difference in the quality of the solutions among the three team compositions can be seen when there is one best solution than multiple best solutions in a design space. Since there are multiple best solutions in the design space with 5 peaks, all the team compositions produce better solution quality than when there was one peak. This difference in performance (when working on a difficult task and a task with multiple alternatives) could be seen most in the all novice agent team.

\subsection{Exploration index}

Figure 7(left) shows the exploration index when agents were generating solutions. It could be seen that the teams with experienced agents in them ( 1 and 3 experienced agents) have lesser exploration index 
when there was only one best solution than multiple best solutions. This is because they had experienced agents who were aware of the failure and success points on the solution space, they consider fewer options than novices (Björklund, 2013). Hence, when the number of best solutions increased, their exploration increased (Chen, 2001). On the other hand, the team of all novice agents did not know about the position of the one best solution on the design space, hence they use 'trial and error' techniques for generating solutions (Ahmed et al., 2003). In the case of a difficult task (i.e., one peak) kept exploring and have a higher exploration index than other team compositions for one peak. On the contrary, the all novice agent team were not aware of the positions or the fact there are multiple solutions so when they find one of the best solutions of the five peaks solution space, they stop exploring other areas and explore lesser than the teams with experienced agents. A similar finding was found in the study done in Christiaans and Dorst, (1992) where some junior students while solving a simple problem did not gather much information as they were unaware of a lot of potential criteria and difficulties on the solution space.
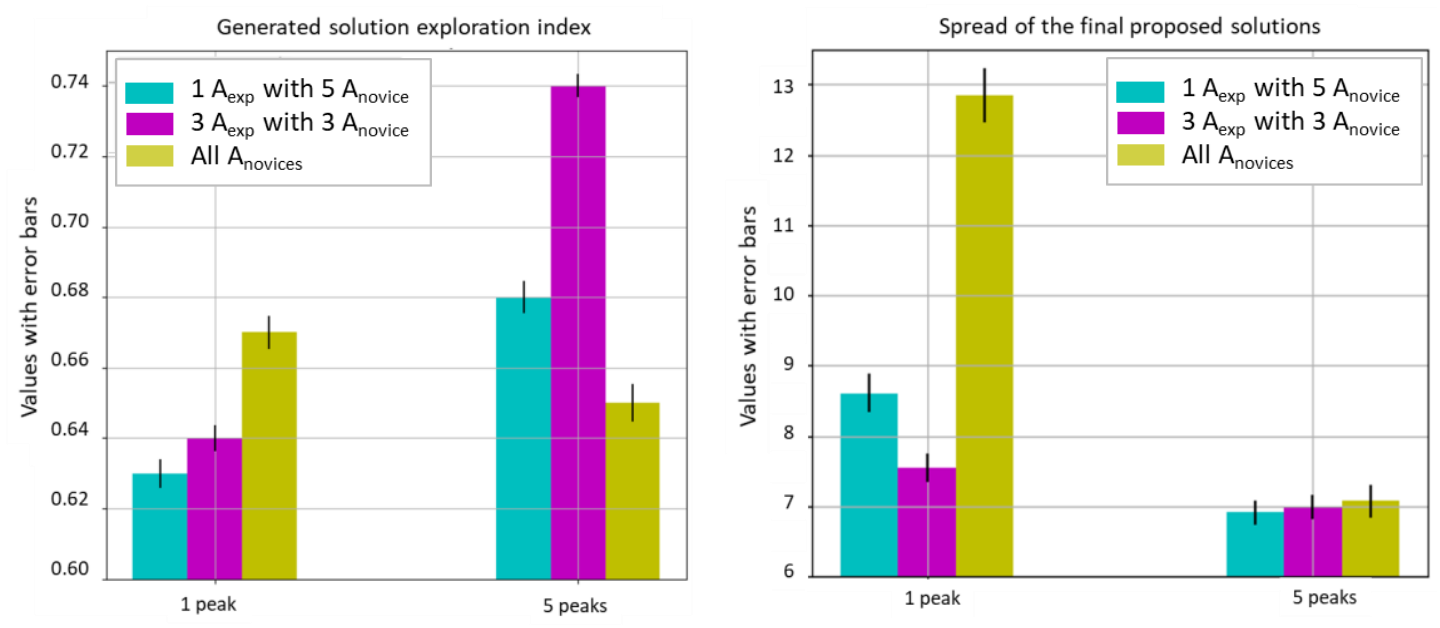

Figure 7.(left) Exploration index of the generated solutions, (right) spread of the final proposed solutions

\subsection{Spread}

Even though the agents in the teams explore the design space while generating solutions, it is not necessary that they have a high variety in their final proposed solutions. An interesting thing to notice in Figure 7(right) is that the spread values for 5 peaks were lesser than one peak design space. In order to understand this, it is important to first know how idea selection works in the model Singh et al. (2020b). Referring to Figure 2 at the beginning of the paper, it can be seen that idea selection starts with communicating solutions by more confident agents and then decisions are made on the proposed solution. Authors like Fricke (1996) explained that when designers produce good early concepts, they need not radically alter them when further exploring solutions, hence good designers have less diversity. Since it is more probable to propose a solution of high quality when there are multiple best solutions, it is easier for those agents proposing the solutions to become even more confident, hence more influential. This makes the same agents to often propose solutions close to their past successful solutions and the rest of the agents agreeing with them due to their influential nature (Singh et al., 2020b).

Figure 7(right) also shows that all novice agent teams had the highest variety in their proposed solutions. As explained above that as the team of all novice agents were not aware of the position or the area of the only best solution present (for one peak design space) so they proposed solutions from all over the design space to the controller agent, hence they the highest variety or spread of their solutions. The team with one experienced agent had more diversity in their solutions than the team with three experienced agents as the degree of influence reduces the variety of solutions.

\section{CONCLUSION}

The findings of the paper are based on the model that considers the previously neglected effects of social factors that emerge when placing novices and experienced in a design team. The results from the model simulation show how different novice-experienced team compositions contribute towards creativity, 
assessed in the form of quality, exploration and variety. Overall, the work shows the impact of different novice-experience team composition on design outcome and results could be interpreted as follows:

- Having experienced individuals in teams with novices could be more useful when the design solutions are difficult to find (i.e., one peak design space).

- It can be seen from the results of the team of all novice agents where they generated high variety and low quality when the task was difficult that conform to studies of engineering design where generating a large variety of solution lead to poor design solutions especially when there are fewer alternatives (one peak design space or difficult to find good solutions). Having a high variety and exploration index in the case of one best solution is not useful as it leads to wastage of resources. Therefore, all novice individual teams working on a project whose design task has fewer alternatives might not be efficient.

- However, in the case of a design problem that has multiple alternative solutions (i.e., five peak design space), any team composition would produce similar variety and quality results.

However, the reliability of the results is subject to the accuracy of the parameters considered in the model. Like the 2-D representation of the solution space where only two aspects of a design problem were considered. Although the work is currently being done on 3 and 4 dimensions of a design space, achieving results for more than 5 dimensions is still computationally exhaustive. Even though the authors have tried to find analogies of the design space representation with the ease and complexity of the conceptual design problem, the design space could not be considered equivalent to very specific to any industrial or innovation projects. The results would vary when very small or large team size is considered. The advantage of the study lies in its simple model that is capable of being represented by some parameters and rules to achieve the given goal. However, more parameters related to the agent, team or task could be added as desired. The work in the future deals with conducting experiments in the real world and using the results to feed and tune the model.

Many of the findings conform to those of the literature, thus partially validating the model. This agentbased model can thus be used with confidence by future researchers to model other aspects of collaborative team design. The work has contributed to the design research in understanding team behaviour of experience-novice team composition. The results could be used to develop suitable team management strategies that managers can use to form a team based on design task and experience level to get better project outcomes.

\section{REFERENCES}

Ahmed, S. and Wallace, K. M. (2004), "Understanding the Knowledge Needs of Novice Designers in the Aerospace Industry”, Design Studies, Vol. 25 No 2, pp. 155-173. https://doi.org/10.1016/j.destud.2003.10.006

Ahmed, S., Wallace, K. M. and Blessing, L. T. (2003), "Understanding the differences between how novice and experienced designers approach design tasks", Research in Engineering Design, Vol. 14, pp. 1-11. https://doi.org/10.1007/s00163-002-0023-z" https://doi.org/10.1007/s00163-002-0023-z

Atman, C. J., Chimka, J. R., Bursic, K. M. and Nachtmann, H. L. (1999), “A Comparison of Freshman and Senior Engineering Design Processes”, Design Studies, Vol. 20 No. 2, pp. 131-152. https://doi.org/10.1016/S0142-694X(98)00031-3

Ball, L. J., Evans, J. S. B. T. and Dennis, I. (1994), “Cognitive processes in engineering design: a longitudinal study”, Special Issue: Cognitive Ergonomics, Vol. 37 No. 11, pp. 1753-1786. https://doi.org/10.1080/00140139408964950

Björklund, . T. A. (2013), "Initial mental representations of design problems: Differences between experts and novices", Design Studies, Vol. 34 No. 2, pp. 135-160. https://doi.org/10.1016/j.destud.2012.08.005

Chamorro-Koc, M., Davis, R. M. and Popovic, V. (2009), "Designers' experience and collaborative design : two case studies”, IASDR2009 Proceedings. Korean Society of Design Science, COEX, Seoul

Chen, S.C. (2001), "The Role of Design Creativity in Computer Media”. Architectural Information Management 19th eCAADe Conference Proceedings, Helsinki (Finland), pp. 226-231.

Christiaans, H.H.C.M. and Dorst, K.H. (1992), "Cognitive models in industrial design engineering: a protocol study", Design theory and methodology, Vol. 42 No 1, pp.131-140.

Cross, N. (2004), "Expertise in Design: an overview", Design Studies, Vol. 25 No. 5, pp. 427-441. https://doi.org/10.1016/j.destud.2004.06.002

Deken, F., Aurisicchio, M., Kleinsmann, M., Lauche, K. and Bracewell, R. (2009), "Novice-Expert Design Consultations: Findings from a Field Study”, ICORD 09: Proceedings of the 2nd International Conference on Research into Design, Bangalore, India, pp. 552-559. 
Deken, F., Kleinsmann, M., Aurisicchio, M., Lauche, K. and Bracewell, R.(2012), “Tapping into past design experiences: knowledge sharing and creation during novice-expert design consultations", Research in Engineering Design, Vol. 23 No.3, pp.203-218. https://doi.org/10.1007/s00163-011-0123-8

Fricke, G. (1996), "Successful individual approaches in engineering design". Research in Engineering Design, Vol. 8 No. 3, pp.151-165.

Fronza, I., Sillitti, A., Succi, G. and Vlasenko, J. (2011), "Understanding how novices are integrated in a team analysing their tool usage", Proceedings of the 2011 International Conference on Software and Systems Process. Honolulu, HI, USA, pp. 204-207. https://doi.org/10.1145/1987875.1987910

Georgilas, I., Dekoninck, E., Dhokia, V., Flynn, J., and Elias, E. (2019), “Comparing different types of professional practitioner engagement in an integrated design engineering degree". DS 95: Proceedings of the 21st International Conference on Engineering and Product Design Education (E\&PDE 2019), University of Strathclyde, Glasgow.

Germain, M.L. (2012), “Traits and Skills Theories as the Nexus between Leadership and Expertise: Reality or Fallacy?", Performance Improvement, Vol. 51 No. 5, pp. 32-39. https://doi.org/10.1002/pfi.21265

Guimerà, R., Uzzi, B., Spiro, J. and Amaral, L. A. (2005). "Team assembly mechanisms determine collaboration network structure and team performance”, Science, New York, pp. 697-702. https://doi.org/10.1126/science. 1106340

Haerem, T. and Rau, D.( 2007), "The influence of degree of expertise and objective task complexity on perceived task complexity and performance," Journal of Applied Psychology, Vol. 92, No. 5, pp. 1320-1331. https://doi.org/10.1037/0021-9010.92.5.1320

Klucharev, V., Smidts, A. and Fernández, G. (2008), "Brain mechanisms of persuasion: how 'expert power' modulates memory and attitudes”, Social Cognitive and Affective Neuroscience, Vol. 3 No. 4, pp. 353-366. https://doi.org/10.1093/scan/nsn022

McComb, C., Cagan, J. and Kotovsky, K. (2015), "Lifting the Veil: Drawing insights about design teams from a cognitively-inspired computational model”, Design Studies, Vol. 40, pp.119-142. https://doi.org/10.1016/j.destud.2015.06.005" https://doi.org/10.1016/j.destud.2015.06.005

Meshi, D., Biele, G., Korn, C. W. and Heekeren, H. R. (2012), "How Expert Advice Influences Decision Making”, PLoS ONE, Vol. 7 No.11, pp. e49748. https://doi.org/10.1371/journal.pone.0049748

Moussaïd, M., Kämmer, J. E., Analytis, P. P. and Neth, H. (2013), "Social influence and the collective dynamics of opinion formation", PLOS ONE, Vol. 8 No.11, pp. e78433.https://doi.org/10.1371/journal.pone.0078433

National Research Council (2000), "How Experts Differ from Novices", In: How People Learn: Brain, Mind, Experience, and School: Expanded Edition, Washington, DC: The National Academies Press, pp. 31-50.

Perisic, M. M., Martinec, T., Storga, M. and Gero, J. S. (2019), “A Computational Study of the Effect of Experience on Problem/Solution Space Exploration in Teams", Proceedings of the 22nd International Conference on Engineering Design (ICED19), Delft, The Netherlands. https://doi.org/10.1017/dsi.2019.4

Singh, H., Cascini, G., Casakin, H. and Singh, V. (2019), "A Computational Framework for Exploring the SocioCognitive Features of Teams and their Influence on Design Outcomes", Proceedings of the 22nd International Conference on Engineering Design (ICED19), Delft, The Netherlands. https://doi.org/10.1017/dsi.2019.3

Singh, H., Cascini, G., and McComb, C. (2020, May), "Analysing The Effect of Self-efficacy and Influencers on Design Team Performance", Proceedings of the Design Society: DESIGN Conference, Cambridge University Press. https://doi.org/10.1017/dsd.2020.64

Singh, H., McComb, C. and Cascini, G. (2020a), "Modelling the Dynamics of Influence on Individual Thinking during Idea Generation in Co-design Teams", Ninth International Conference on Design Computing and Cognition (DCC20), Springer

Singh, H., Cascini, G. and McComb, C.(2020b), "Idea Selection in Collaborative Design Teams: A Computational Framework and Insights in the Presence of Influencers", Design Science (under review)

Singh, V. and Casakin, H. (2018), "Use of Analogy in Design Teams: Steps towards a computational model and conceptual insights", Proceedings of the DESIGN 2018 15th International Design Conference, Dubrovnik, Croatia, https://doi.org/10.21278/idc.2018.0342

Wang, Z. (2018), “The Impact of Expertise on Pair Programming Productivity in a Scrum Team: A Multi-Agent Simulation", IEEE 9th International Conference on Software Engineering and Service Science (ICSESS), Beijing, China, pp. 399-402.10.1109/ICSESS.2018.8663874.

Zaki, J., Schirmer, J. and Mitchel, J. P. (2011), "Social Influence Modulates the Neural Computation of Value", Psychological Science, Vol. 22 No.7, pp. 894-900. https://doi.org/10.1177/0956797611411057

Zeiler, W.(2013), "Cooperation between novice designers (students) and professional in building industry", Proceedings of E\&PDE 2013, the 15th International Conference on Engineering and Product Design Education, Dublin, Ireland. 